\title{
Análise de opinião referente ao uso de ferramentas digitais por comerciantes do município de Tefé-AM
}

Opinion analysis regarding the use of digital tools by merchants in the municipality of Tefé-AM Análisis de opinión sobre el uso de herramientas digitales por comerciantes del municipio de Tefé-

Rafael Bernhard

ORCID: https://orcid.org/0000-0001-5435-8073 Universidade do Estado do Amazonas, Brasil E-mail: rafbernhard@gmail.com

Eloá Arevalo Gomes Fraga

ORCID: https://orcid.org/0000-0003-0097-1002 Universidade do Estado do Amazonas, Brasil E-mail: eagomes@uea.edu.br

Elzalina Ribeiro Soares

ORCID: https://orcid.org/0000-0002-9267-8841 Universidade do Estado do Amazonas, Brasil E-mail: ersoares@uea.edu.br

Crystiane Teresa Vieira Frazão ORCID: https://orcid.org/0000-0001-7312-6550 Universidade do Estado do Amazonas, Brasil E-mail: ctvf.qui16@uea.edu.br

Vitor Mateus Daniel da Costa ORCID: https://orcid.org/0000-0002-1561-5636 Universidade do Estado do Amazonas, Brasil E-mail: vmddc.his17@uea.edu.br

Ingrid Bianca Ferreira Da Silva ORCID: https://orcid.org/0000-0001-9485-4549 Universidade do Estado do Amazonas, Brasil E-mail: ibfs.bio17@uea.edu.br Geise Noteno Moura ORCID: https://orcid.org/0000-0001-8128-855X Universidade do Estado do Amazonas, Brasil E-mail: geisenotenobio@gmail.com Alessandra Pinto da Silva ORCID: https://orcid.org/0000-0003-1747-5953 Universidade do Estado do Amazonas, Brasil E-mail: aps.bio17@uea.edu.br Alisson Nogueira Cruz ORCID: https://orcid.org/0000-0002-6674-880X Universidade do Estado do Amazonas, Brasil

E-mail: alissonnogueiracruz@gmail.com Jackson Leno da Silva Monteiro ORCID: https://orcid.org/0000-0002-0235-0595 Universidade do Estado do Amazonas, Brasil E-mail: jldsm.qui16@uea.edu.br Messias Dias Clarindo

ORCID: https://orcid.org/0000-0002-6354-9215 Universidade do Estado do Amazonas, Brasil E-mail:mdc.bio16@uea.edu.br

Moises Dias Clarindo

ORCID: https://orcid.org/0000-0003-2532-1161 Universidade do Estado do Amazonas, Brasil E-mail:mdc.bio16@uea.edu.br Willison Pinto da Silva

ORCID: https://orcid.org/0000-0001-7202-2936 Universidade do Estado do Amazonas, Brasil E-mail: wpds.mat21@uea.edu.br

Silvia Regina Sampaio Freitas ORCID: https://orcid.org/0000-0003-2987-7837 Universidade do Estado do Amazonas, Brasil 


\begin{abstract}
Resumo
O mundo vem passando por diversas transformações digitais, e a aplicação de novas tecnologias para criação de moedas sociais e plataformas financeiras é algo que vem difundindo-se entre a população de baixa renda, devido a popularização da internet, por ser uma saída mais lucrativa e segura, evitando o acúmulo de dinheiro em forma física. Com a criação de novas tecnologias, vem crescendo a imposição de padrões para quem é usuário de Smartphone, cada vez mais as empresas estão desenvolvendo aplicativos com o seu formato de uso. No entanto, fatores como a diversidade geográfica torna-se um grande desafio no processo de popularizar o uso das tecnologias de rede no estado do Amazonas, uma vez que muitos lugares são de difícil acesso. Assim, o objetivo do trabalho foi realizar uma pesquisa de opinião dos comerciantes do munícipio de Tefé - AM sobre o uso de internet, formas de pagamento utilizadas pela população, além do conhecimento e adesão relacionada ao uso de uma moeda social e plataformas eletrônicas digitais para esse fim. A metodologia utilizada foi uma entrevista realizada de forma presencial em 12 categorias de comércios divididos em 19 bairros. Os dados coletados foram inseridos no banco de dados do Google Forms e posteriormente realizadas análises através de tabelas e gráficos. Ao final da pesquisa verificou-se que o uso de aplicativos para transações financeiras ainda é baixo, mas há influência positiva que a tecnologia de uma plataforma financeira irá proporcionar nos comerciantes do município de Tefé.
\end{abstract}

Palavras-chave: Plataformas digitais; Moeda social; E-commerce Brasil; Transformação digital.

\begin{abstract}
The world has been undergoing several digital transformations, and the application of new technologies for the creation of social currencies and financial platforms is something that has been spreading among the low-income population, due to the popularization of the Internet, because it is a more profitable exit and safer, avoiding the accumulation of money in physical form. With the creation of new technologies, the imposition of standards for smartphone users is growing, more and more companies are developing applications with their usage format. However, factors such as geographic diversity becomes a major challenge in the process of popularizing the use of network technologies in the state of Amazonas, since many places are difficult to access. Thus, the objective of this work was to conduct an opinion survey of traders in the municipality of Tefé -Am on the use of internet, payment method used by the population, in addition to knowledge and adhering related to the use of a social currency and digital electronic platforms for this purpose. The methodology used was an interview conducted in person in 12 categories of trades divided into 19 neighborhoods. The collected data were entered in the Google Forms database and later performed analysis through tables and graphs. At the end of the research it was found that the use of applications for financial transactions is still low, but there is positive influence that the technology of a financial platform will provide in traders of the municipality of Tefé.
\end{abstract}

Keywords: Digital platforms; Social currency; E-commerce Brasil; Digital transformation.

\title{
Resumen
}

El mundo ha estado experimentando varias transformaciones digitales, y la aplicación de nuevas tecnologías para la creación de monedas sociales y plataformas financieras es algo que se ha ido extendiendo entre la población de bajos ingresos, debido a la popularización de Internet, porque es una salida más rentable, y segura, evitando la acumulación de dinero en forma física. Con la creación de nuevas tecnologías, la imposición de estándares para los usuarios de teléfonos inteligentes está creciendo, cada vez más empresas están desarrollando aplicaciones con su formato de uso. Sin embargo, factores como la diversidad geográfica se convierten en un gran desafío en el proceso de popularización del uso de tecnologías de red en el estado de Amazonas, ya que muchos lugares son de difícil acceso. Así, el objetivo de este trabajo fue realizar una encuesta de opinión a comerciantes del municipio de Tefé-Am sobre el uso de internet, método de pago utilizado por la población, además de conocimientos y adherencias relacionadas con el uso de una moneda social y plataformas electrónicas digitales para tal fin. La metodología utilizada fue una entrevista realizada de manera presencial en 12 categorías de oficios divididos en 19 barrios. Los datos recogidos se introdujeron en la base de datos de Google Forms y posteriormente se realizaron análisis a través de tablas y gráficos. Al final de la investigación se encontró que el uso de aplicaciones para transacciones financieras sigue siendo bajo, pero hay una influencia positiva que la tecnología de una plataforma financiera proporcionará en los comerciantes del municipio de Tefé.

Palabras clave: Plataformas digitales; Moneda social; E-commerce Brasil; Transformación digital.

\section{Introdução}

$\mathrm{O}$ acesso à internet deixou de ser restrito a uma parte das pessoas e atualmente tornou-se comum no cotidiano de grande parcela da população mundial (Guidini, 2018). A evolução da tecnologia revolucionou o tipo de comunicação na sociedade impactando profundamente as relações comerciais modificando os modelos tradicionais de negócios (Diniz, 1999; Correa et al., 2006; Guidini, 2018).

Com os avanços tecnológicos no início na década de 90 e a propagação da internet, houve uma mudança no 
comportamento dos consumidores e as empresas precisaram adaptar-se a esses novos desafios e passaram a utilizar o comércio eletrônico (e-commerce) com cada vez mais recursos eletrônicos em seus negócios, ganhando rapidez, eficiência e considerável redução de custos (Yoshida, 2015; Bez Fontana et al., 2018; Freire \& Salgado, 2019).

Esse crescimento da internet foi primordial para a expansão do e-commerce no mundo, tornando-se um comércio indispensável pela maioria de seus adeptos e usuários. Com o avanço de novas tecnologias, a imposição relacionada a padrões de uso para os usuários de Smartphone vem crescendo, e as empresas cada vez mais estão desenvolvendo aplicativos com o formato de uso que mais se adeque a vertente do mercado e da empresa. Como exemplo, tem-se os navegadores usados por muito tempo, Firefox e Chrome, esses eram os programas principais para utilizar os "sites" da Internet. Por meio do crescimento tecnológico é possível utilizar formas mais simples de acesso à internet, como pelos aplicativos, que proporcionam aos seus usuários acesso mais rápido e direto. Isso se dá pelo fato que de as empresas estão se adaptando às novas tecnologias digitais (Chew, 2015; Okano et al., 2019).

No Brasil, o comércio pela internet começou no ano 2000, voltado a ampliação ao lojas varejistas como Americanas, Submarino e o grupo Pão-de-Açúcar (Viana, 2014; Bez Fontana et al., 2018). Segundo a Global Payments Report - Worldpay (2018), o gasto por meio de comércio eletrônico no Brasil foi de 116\$ per capita em 2017 com projeção para crescimento de $6 \%$ até 2022, enquanto para o comércio em loja física é de 5\%, o que mostra uma preferência pelo ambiente virtual. No entanto, com a pandemia do Sars-Cov2 essa projeção foi modificada. De acordo com Ebit/Nielsen (2021) o comércio eletrônico subiu $41 \%$ em relação a 2019, em compras realizadas principalmente por celular. Apesar do e-commerce ser ainda um desafio no Brasil seja pela internet instável em alguns locais que prejudicam a finalização das compras, seja por problemas de logística (Alves \& Chaves, et al., 2005; Freire \& Salgado, 2019), a interiorização do e-commerce acelerou por conta da pandemia com um aumento de $18,7 \%$ na região nordeste (Ebit/Nielsen (2021).

Conforme dados do Sebrae (2017), o acesso à internet via dispositivo móvel foi de 52,4\% no ano de 2015, e no ano de 2019 a região norte foi a que mais utilizou o serviço de banda larga móvel, sendo esta utilizada por 88,6\% da população. (AgênciaBrasil, 2021). Todavia a disseminação do uso de tecnologias enfrenta dificuldades como a diversidade geográfica, visto que estas dificultam a implementação de redes de comunicação, necessárias para a utilização destas novas tecnologias em municípios do interior.

Uma das cidades do estado do Amazonas que enfrenta esta dificuldade é o município de Tefé. O acesso ao município se dá por vias fluviais e aérea (Santos, 2012). Esta cidade é considerada uma das mais antigas do estado, sendo o seu registro entre os anos de 1686 e 1688 e de acordo com Ribeiro (1996) sendo considerada importante entreposto comercial do Médio Solimões (Rodrigues, 2011).

Diante desse panorama, o objetivo do presente trabalho foi caracterizar o perfil do comerciante e do comércio local, analisar as formas de pagamento utilizadas no comércio, bem como, a percepção que os comerciantes têm sobre a qualidade de acesso à internet e verificar a aceitabilidade do uso de plataformas digitais eletrônicas e moedas sociais.

\section{Metodologia}

A presente pesquisa foi realizada na cidade de Tefé, no estado do Amazonas, localizada entre as coordenadas de $03^{\circ}$ $15^{\prime} 39^{\prime \prime}$ a $05^{\circ} 34^{\prime} 22^{\prime \prime}$ de latitude Sul e $64^{\circ} 04^{\prime} 12^{\prime \prime}$ a $68^{\circ} 58^{\prime} 32^{\prime \prime}$ de longitude oeste com extensão territorial aproximadamente de $23.704 \mathrm{~km}^{2}$ (Rodrigues, 2011). A população do município, de acordo com o último censo do IBGE em 2010, era de 61.543 habitantes, sendo que destes, 50.069 eram da área urbana (IBGE, 2021). Para este artigo foram entrevistados 121 empresários entre os dias 8 e 20 de julho de 2021, onde foi exposto aos entrevistados o objetivo do estudo e assegurado sua anonimidade. Todas as entrevistas foram realizadas de forma voluntária em 19 dos 22 bairros da cidade.

A metodologia utilizada foi a pesquisa por sondagem ou survey na qual obtêm-se dados perguntando-se às pessoas 
através de um questionário estruturado sobre características, ações ou opiniões (Pinsonneault \& Kraemer, 1993; Hair et al., 2003) e tem por objetivo produzir estatísticas, porcentagens sobre algum aspecto dessa população (Fowler, 2009)

Para a pesquisa foi elaborado um instrumento de coleta de dados em forma de questionário semiestruturado por meio de formulário eletrônico contendo 15 perguntas abertas e fechadas abordando uma prévia caracterização dos comerciantes (idade e gênero) e dos comércios (categoria e número de funcionários), os principais meios utilizados para receber pagamento dos seus produtos, como realizam pagamentos, se conhecem plataformas de comércio eletrônico e se teriam interesse em utilizar uma plataforma relacionada a esse fim para anunciar seus produtos e se já ouviram sobre moeda social (Apêndice 1). Caso o entrevistado tenha declarado desconhecimento sobre moeda social, o entrevistador realizava uma breve explicação sobre este termo e seu significado antes da última pergunta, acerca da disposição do entrevistado em utilizá-la.

Foi calculada a média, desvio padrão (DP) e indicado valor máximo e mínimo para as respostas. Para testar se houve diferença nas respostas entre os gêneros masculino e feminino foi utilizada teste de X2 de Pearson para aderência com $\alpha=5 \%$ (Vieira, 2016). Para esse teste as respostas não souberam ou não quiseram responder foram excluídas.

\section{Resultados e Discussão}

Dentre os 19 bairros visitados, o Centro concentrou o maior número de entrevistas ( $\mathrm{N}=52)$, seguido do bairro Juruá $(\mathrm{N}=14)$ e Bom Jardim Lara ( $\mathrm{N}=8$ ). Nos demais bairros, o número de entrevistas variou entre um e cinco (Figura 1), pois apesar de serem bairros populosos o Centro concentra estabelecimentos de grande circulação de pessoas, como bancos, escolas e o porto da cidade. Enquanto o bairro Juruá contém vias importantes de deslocamento da população e por isso é mais interessante para os comerciantes.

Entre os 121 comerciantes entrevistados, 54 (44,6\%) foram do gênero feminino com média de idade de 37 anos (DP = 10; 20-63), 66 (54,5\%) do gênero masculino com idade média de 35 anos ( $\mathrm{DP}=11 ; 19-74)$ e $1(0,8 \%)$ que se declarou de outro gênero com 35 anos de idade.

As categorias de comércio observadas no município estão apresentadas na Tabela 1. As entrevistas foram realizadas em 12 tipos de comércio, muito comuns na cidade. Destacou-se a categoria de varejo pequeno com 47 estabelecimentos, seguido de lanchonetes, varejo médio e distribuidoras. A maior quantidade de estabelecimentos de varejo pequeno pode ocorrer devido a facilidade de caracterização desta categoria, pois inclui mercearias que são localizadas, muitas vezes, nas próprias residências dos comerciantes. Em relação à quantidade média de funcionários por estabelecimento comercial foi de 4,43 variando de zero (quando existia apenas o dono do estabelecimento) a 52 funcionários em um varejo grande (Tabela 1). 
Figura 1. Distribuição das entrevistas aos comerciantes nos bairros de Tefé (AM). Centro e Centro A foram agrupados para a análise pois representam apenas um bairro para fins práticos. O bairro do aeroporto não consta no shapefile, este foi representado por um polígono em uma área aproximada de sua ocorrência.

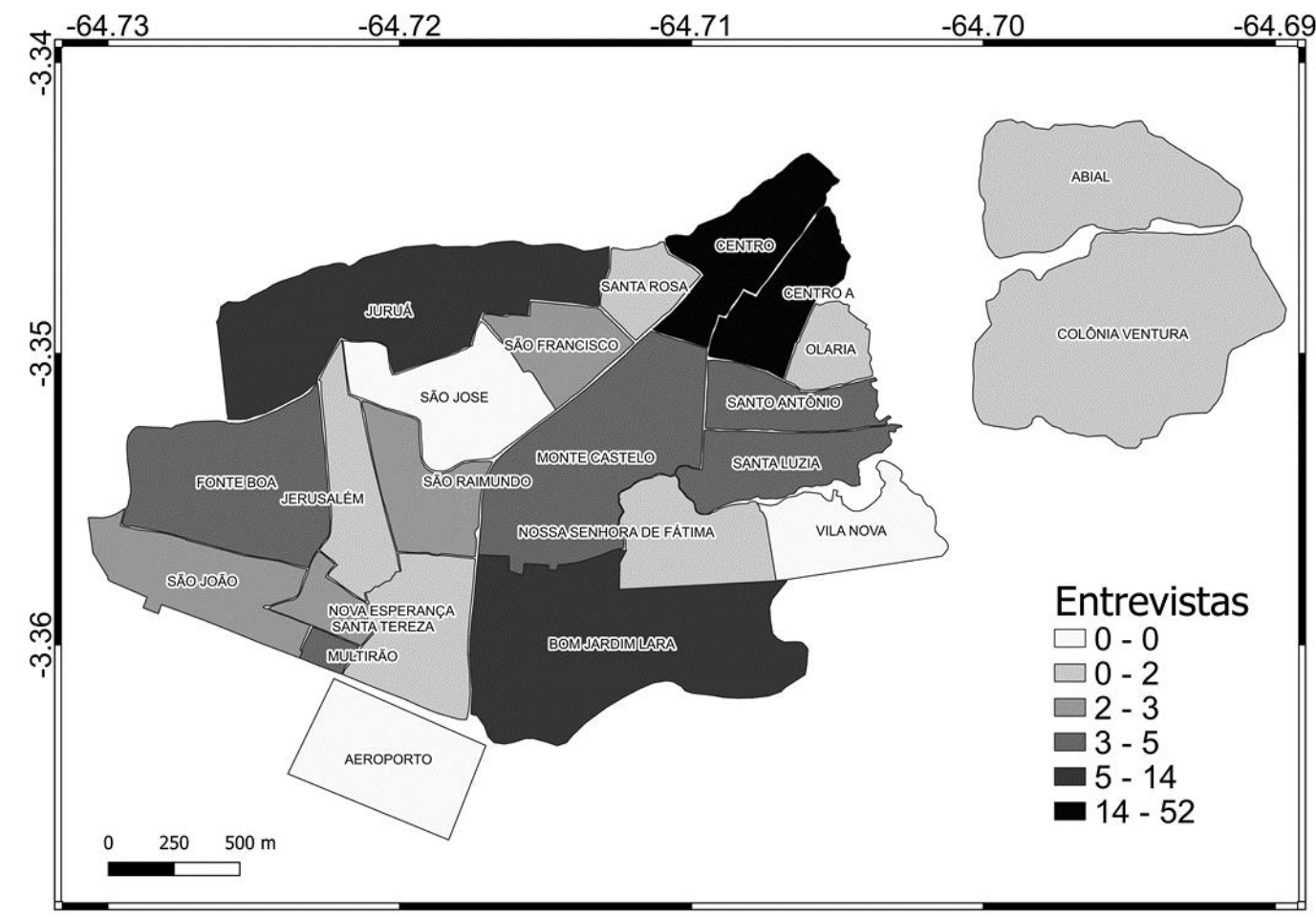

Fonte: Mapa elaborado pelos autores utilizando o shapefile desenvolvido pelo IDSM (2021). Datum WGS 72/UTM zona 20S (EPSG: 32320).

Tabela 1. Número de funcionários nos diferentes tipos de comércios de Tefé (AM), valores médios, mínimos e máximos. N: número de comércios amostrados por categoria.

\begin{tabular}{lcccc}
\hline \multirow{2}{*}{ Tipo de comércio } & \multicolumn{4}{c}{ Quantidade de funcionários } \\
\cline { 2 - 5 } & $\mathrm{N}$ & Média & Mínimo & Máximo \\
\hline Varejo grande & 6 & 24.67 & 12 & 52 \\
Varejo médio & 15 & 3.93 & 2 & 7 \\
Varejo pequeno & 47 & 2.02 & 0 & 6 \\
Padaria & 6 & 6.33 & 1 & 20 \\
Lanchonete & 15 & 3.20 & 1 & 8 \\
Hortifruti & 1 & 3.00 & 3 & 3 \\
Distribuidora & 9 & 6.00 & 1 & 19 \\
Farmácia & 5 & 7.60 & 3 & 15 \\
Material de construção & 3 & 3.67 & 2 & 6 \\
Celulares e eletrônicos & 5 & 3.40 & 2 & 5 \\
Posto de combustivel & 1 & 10.00 & 10 & 10 \\
Moda e calçado & 7 & 1.57 & 0 & 4 \\
\hline Total & 120 & 4.43 & 0 & 52 \\
\hline
\end{tabular}

Fonte: Autores.

Em relação as formas de pagamento que os comerciantes dispõem, o dinheiro representa a grande preferência (95\%), seguido do cartão de crédito $(29,8 \%)$ e do Pix $(25,6 \%)$ (Tabela 2). No entanto, apesar de haver várias formas para receber o pagamento dos seus produtos, ainda prevalece o recebimento em dinheiro $(96,7 \%)$, seguido por cartão de crédito e débito ( $8,3 \%$ e $7,4 \%$ respectivamente) (Tabela 3 ). A caderneta consiste em um método de pagamento muito utilizado na região no passado, mas que não foi relatada durante a pesquisa. Apesar das dificuldades de troco relatadas pelos comerciantes, o mau 
funcionamento das máquinas de cartão para viabilizar outras formas de pagamento pode influenciar a maior ocorrência das vendas em dinheiro como observado nos resultados da pesquisa.

Tabela 2. Formas de pagamento aceitas pelos comerciantes de Tefé (AM). Cada entrevistado pode ter mencionado mais de uma forma de pagamento.

\begin{tabular}{lcc}
\hline Forma de pagamento & $\mathrm{N}$ & $\%$ \\
\hline Dinheiro & 115 & 95,0 \\
Cartão de crédito & 36 & 29,8 \\
Pix & 31 & 25,6 \\
Cartão de débito & 28 & 23,1 \\
Promissória & 3 & 2,5 \\
DOC/TED & 3 & 2,5 \\
Caderneta & 0 & 0,0 \\
Outros & 7 & 5,8 \\
Não quis responder & 5 & 4,1 \\
\hline
\end{tabular}

Fonte: Autores.

Tabela 3. Formas de pagamento com as quais os comerciantes mais declaram receber pelos produtos em Tefé (AM). Cada entrevistado pode ter mencionado mais de uma forma de pagamento.

\begin{tabular}{lcc}
\hline Forma de pagamento & $\mathrm{N}$ & $\%$ \\
\hline Dinheiro & 117 & 96,7 \\
Cartão de crédito & 10 & 8,3 \\
Cartão de débito & 9 & 7,4 \\
Pix & 5 & 4,1 \\
Promissória & 1 & 0,8 \\
DOC/TED & 0 & 0,0 \\
Caderneta & 0 & 0,0 \\
Outros & 5 & 4,1 \\
Não quis responder & 0 & 0,0 \\
\hline
\end{tabular}

Fonte: Autores.

Mesmo apresentando um baixo número de transações na cidade de Tefé, o Pix está atrelado a uma mudança de cenário do comércio brasileiro. Lima \& Francisco (2021) relatam essa mudança de cenário nos meios de pagamentos digitais e que deve mudar muito nos próximos anos, levando em consideração o modo como a população está e irá se comportar perante a pandemia da Covid-19 e o lançamento e crescimento dos pagamentos por Pix.

Ao serem questionados onde realizam seus pagamentos, os comerciantes relataram que de maneira geral pagam nas casas lotéricas $(46,3 \%)$, nos bancos, com os caixas $(40 \%)$ e por aplicativo de celular $(33,9 \%)$. Outros métodos de pagamento mencionados foram o Pix (duas vezes), pagamento diretamente para o fornecedor (uma vez) ou realizado pela loja central (duas vezes) (Tabela 4). 
Tabela 4. Formas utilizadas pelos comerciantes de Tefé (AM) para pagar as suas contas.

\begin{tabular}{lcc}
\hline Forma de pagar contas & $\mathrm{N}$ & $\%$ \\
\hline Lotérica & 56 & 46,3 \\
Fila de banco & 49 & 40,5 \\
Aplicativo de celular & 41 & 33,9 \\
Caixa eletrônico & 29 & 24,0 \\
Correios & 5 & 4,1 \\
Outros & 5 & 4,1 \\
Não quis responder & 2 & 1,7 \\
\hline
\end{tabular}

Fonte: Autores.

Com a pandemia da Covid-19, o comércio em muitos momentos foi fechado na cidade, assim como o deslocamento via área e fluvial foi proibido, impossibilitando a vinda de consumidores de municípios vizinhos e comunidades e também o escoamento de bens e serviços. Mesmo com as limitações apresentadas os comércios na cidade continuaram a receber o pagamento majoritariamente em papel moeda e formas de pagamento como cartões de crédito e Pix foram minorias nesta pesquisa, diferentemente do que foi apresentado na pesquisa da EY Consultoria em que relata que os meios de pagamentos de forma digital aumentaram cerca de $43 \%$ após o início da pandemia no ano de 2020 (Lima \& Francisco, 2020)

A maioria dos comerciantes $(\mathrm{N}=69 ; 57 \%)$ declarou ter aplicativos para pagamento no celular, no entanto, $48(39,7 \%)$ declararam não ter. Quatro comerciantes não quiseram responder a esta questão. Dentre os que possuem algum aplicativo, a maioria paga pelos aplicativos de banco convencionais (54,5\%) enquanto o Nubank aparece como segunda opção mais utilizada com 7,4\% (Tabela 5).

Tabela 5. Aplicativos de pagamentos utilizados pelos comerciantes em Tefé (AM) em seus celulares.

\begin{tabular}{lcc}
\hline Aplicativos & $\mathrm{N}$ & $\%$ \\
\hline Aplicativos de bancos & 66 & 54,5 \\
Nubank & 9 & 7,4 \\
PicPay & 5 & 4,1 \\
PayPal & 2 & 1,7 \\
Next & 2 & 1,7 \\
Pague Seguro & 1 & 0,8 \\
Mercado Pago & 1 & 0,8 \\
Nenhum & 47 & 38,8 \\
\hline
\end{tabular}

Fonte: Autores.

Apesar dos resultados mostrarem a utilização de aplicativos, apenas 23,1\% dos comerciantes estão satisfeitos com os serviços de internet oferecidos na cidade. A maioria das respostas $(72,7 \%)$ demonstrou algum grau de insatisfação sem diferença entre homens e mulheres $\left.\left(\mathrm{X}_{(\mathrm{g} . \mathrm{ll}}^{2}=4, \mathrm{~N}=119\right)=1,289 ; p=0,86\right)$ (Tabela 6). 
Tabela 6. Grau de satisfação dos comerciantes de Tefé (AM) com a qualidade da internet da cidade.

\begin{tabular}{|c|c|c|c|c|c|c|c|}
\hline \multirow{3}{*}{$\begin{array}{c}\text { Grau de satisfação } \\
\text { Plenamente satisfeito }\end{array}$} & \multicolumn{5}{|c|}{ Gênero } & \multirow{2}{*}{\multicolumn{2}{|c|}{ Total }} \\
\hline & \multicolumn{2}{|c|}{ Feminino } & \multicolumn{2}{|c|}{ Masculino } & \multirow{2}{*}{$\begin{array}{r}\text { Outro } \\
0\end{array}$} & & \\
\hline & 2 & $3,7 \%$ & 2 & $3,0 \%$ & & 4 & $3,3 \%$ \\
\hline Apenas satisfeito & 10 & $18,5 \%$ & 14 & $21,2 \%$ & 0 & 24 & $19,8 \%$ \\
\hline Insatisfeito & 20 & $37,0 \%$ & 30 & $45,5 \%$ & 1 & 51 & $42,1 \%$ \\
\hline Muito insatisfeito & 19 & $35,2 \%$ & 18 & $27,3 \%$ & 0 & 37 & $30,6 \%$ \\
\hline Não quis responder & 1 & $1,9 \%$ & 0 & $0,0 \%$ & 0 & 1 & $0,8 \%$ \\
\hline Não tenho opinião & 2 & $3,7 \%$ & 2 & $3,0 \%$ & 0 & 4 & $3,3 \%$ \\
\hline Total & 54 & $100,0 \%$ & 66 & $100,0 \%$ & 1 & 121 & $100,0 \%$ \\
\hline
\end{tabular}

Fonte: Autores.

O comércio de forma presencial foi reduzido em todo o Brasil a partir de 2020 com a declaração da OMS sobre a pandemia do Covid-19, assim, comprar em plataformas pela internet tornou-se uma alternativa necessária (Premebida, 2021). O comércio eletrônico em Tefé ainda é incipiente principalmente por causa do sinal disponível da internet, como foi possível observar com o questionário realizado neste artigo. No entanto, o uso de uma plataforma eletrônica paga não foi descartada a partir de mais informações sobre custos e como usar.

Garcia e colaboradores (2020), relataram que a Associação Brasileira de Comércio Eletrônico, mostrou que mesmo com a pandemia há tendência de crescimento do e-commerce, pois é dessa forma que os comerciantes continuam se mantendo no mercado, mas segundo Guidini (2018), para garantir as compras, os consumidores preferem aplicativos que tenham uma linguagem funcional e o acesso seja prazeroso.

Okano e colaboradores (2019) apresentam um resultado sobre os apps e as plataformas digitais, podendo comprovar na pesquisa que a $64 \%$ dos entrevistados não tem dificuldades para usar as novas tecnologias porque são elaboradas de forma simples e auto explicativa.

Apesar dos comerciantes de Tefé relatarem que usam poucos aplicativos para fazerem seus pagamentos e não usam Plataformas eletrônicas para venderem seus produtos, o e-commerce é um canal para aumentar as vendas em diversas situações como é o caso da pandemia, pois reduz custos e o consumidor não precisa se deslocar até a loja física, e pode comprar a qualquer hora do dia (Garcia et al., 2020).

Não houve diferença significativa nas respostas entre homens e mulheres quanto ao grau de interesse em utilizar uma plataforma online para anunciar produtos $\left(\mathrm{X}_{(\mathrm{g} .1 \mathrm{l}=3, \mathrm{~N}=116)}^{2}=4,672 ; p=0,20\right)$ embora o percentual de mulheres que tenham se mostrado muito interessadas tenha sido maior do que o dos homens (Tabela 7). Grande e médio interesse representaram a maior parte das respostas $(74,3 \%)$.

Embora haja um interesse inicial baixo em utilizar uma plataforma digital paga $(8,3 \%)$, se o valor for apropriado 47,1\% dos comerciantes se dispõem a utilizá-la (Tabela 8). Quando consideramos o número médio de funcionários por estabelecimento, aparentemente os comércios com 2,8 (DP = 1,96) funcionários em média não querem uma plataforma paga, enquanto comércios com 5,1 ( $\mathrm{DP}=5,24)$ funcionários em média responderam que querem e com 4,2 (DP = 4,07) funcionários condicionaram a aceitação da plataforma ao preço cobrado e não houve diferença nas respostas entre homens e mulheres $\left(\mathrm{X}^{2}\right.$ (g.l. $=2, \mathrm{~N}=109)=1,396 ; p=0,50)$. 
Tabela 7. Grau de interesse dos comerciantes em anunciar os seus produtos em uma plataforma online.

\begin{tabular}{|c|c|c|c|c|c|c|c|}
\hline \multirow{2}{*}{$\begin{array}{c}\text { Você gostaria de } \\
\text { anunciar seus produtos } \\
\text { em uma plataforma } \\
\text { online? }\end{array}$} & \multicolumn{5}{|c|}{ Gênero } & \multirow{2}{*}{\multicolumn{2}{|c|}{ Total }} \\
\hline & \multicolumn{2}{|c|}{ Feminino } & \multicolumn{2}{|c|}{ Masculino } & Outro & & \\
\hline Muito & 24 & $44,4 \%$ & 18 & $27,3 \%$ & 1 & 43 & $35,5 \%$ \\
\hline Talvez & 20 & $37,0 \%$ & 27 & $40,9 \%$ & 0 & 47 & $38,8 \%$ \\
\hline Pouco & 3 & $5,6 \%$ & 9 & $13,6 \%$ & 0 & 12 & $9,9 \%$ \\
\hline De forma alguma & 6 & $11,1 \%$ & 9 & $13,6 \%$ & 0 & 15 & $12,4 \%$ \\
\hline Não quis responder & 1 & $1,9 \%$ & 3 & $4,5 \%$ & 0 & 4 & $3,3 \%$ \\
\hline Total & 54 & $100,0 \%$ & 66 & $100,0 \%$ & 1 & 121 & $100,0 \%$ \\
\hline
\end{tabular}

Fonte: Autores.

Tabela 8. Grau de interesse dos comerciantes de Tefé (AM) em utilizar uma plataforma paga.

\begin{tabular}{lrrrrrrrr}
\hline \multirow{2}{*}{$\begin{array}{c}\text { Se essa plataforma } \\
\text { fosse paga você teria } \\
\text { interesse em utilizar? }\end{array}$} & \multicolumn{9}{c}{ Feminino } & \multicolumn{2}{c}{ Masculino } & Outro & & \\
\cline { 2 - 7 } Sim & 6 & $11,1 \%$ & 4 & $6,1 \%$ & 0 & 10 & $8,3 \%$ \\
Talvez, depende do valor & 25 & $46,3 \%$ & 31 & $47,0 \%$ & 1 & 57 & $47,1 \%$ \\
Não & 17 & $31,5 \%$ & 26 & $39,4 \%$ & 0 & 43 & $35,5 \%$ \\
Não quis responder & 6 & $11,1 \%$ & 5 & $7,6 \%$ & 0 & 11 & $9,1 \%$ \\
\hline Total & 54 & $100,0 \%$ & 66 & $100,0 \%$ & 1 & 121 & $100,0 \%$ \\
\hline
\end{tabular}

Fonte: Autores.

Em relação ao conhecimento sobre a moeda social, ficou evidente a falta de informação dos comerciantes locais, tendo em vista que menos de $2 \%$ dos comerciantes de ambos os gêneros entendem tudo sobre moeda social (Tabela 9) e não houve diferença nas respostas entre homens e mulheres $\left.\left(X_{(\mathrm{g} .1 .}^{2}=4, N=120\right)=0,788 ; p=0,94\right)$.

Tabela 9. Conhecimento dos comerciantes sobre moeda social.

\begin{tabular}{lrrrrrrr}
\hline \multicolumn{1}{c}{$\begin{array}{c}\text { Você sabe o que é uma } \\
\text { moeda social? }\end{array}$} & \multicolumn{4}{c}{ Geminino } & \multicolumn{2}{c}{ Masculino } & \multicolumn{2}{c}{ Outro } & \multirow{2}{*}{ Total } \\
\cline { 2 - 6 } & 1 & $1,9 \%$ & 1 & $1,5 \%$ & 0 & 2 & $1,7 \%$ \\
\hline Entendo tudo sobre ela & 1 & $1,9 \%$ & 1 & $1,5 \%$ & 0 & 2 & $1,7 \%$ \\
Entendo razoavelmente bem & 2 & $3,7 \%$ & 4 & $6,1 \%$ & 0 & 6 & $5,0 \%$ \\
Entendo um pouco & 4 & $7,4 \%$ & 7 & $10,6 \%$ & 0 & 11 & $9,1 \%$ \\
Só ouvi falar & 46 & $85,2 \%$ & 53 & $80,3 \%$ & 1 & 100 & $82,6 \%$ \\
Nem ouvi falar & 54 & $100,0 \%$ & 66 & $100,0 \%$ & 1 & 121 & $100,0 \%$ \\
\hline Total & & & & & & &
\end{tabular}

Fonte: Autores.

No quesito sobre utilizar uma nova forma de recebimento, novamente os comerciantes do gênero feminino possuem um interesse um pouco maior em adquirir uma nova plataforma com total de $64,8 \%$ em relação ao gênero masculino com $51,5 \%$. Um fato motivador que apenas 2,5\% dos comerciantes informaram que não aceitariam uma nova forma de recebimentos por suas mercadorias (tabela 10). E não houve diferença nas respostas entre homens e mulheres $\left(X^{2}{ }_{(\mathrm{g} .1 .}=4, N=118\right)=$ $3,368 ; p=0,50)$. 
Tabela 10. Interesse dos comerciantes de Tefé (AM) em utilizar uma nova ferramenta para o recebimento pela venda dos seus produtos.

\begin{tabular}{lrrrrrrr}
\hline \multirow{2}{*}{$\begin{array}{c}\text { Interesse em utilizar a } \\
\text { plataforma }\end{array}$} & \multicolumn{4}{c}{ Geminino } & \multicolumn{1}{c}{ Masculino } & Outro & \multicolumn{2}{c}{ Total } \\
\cline { 2 - 7 } & 35 & $64,8 \%$ & 34 & $51,5 \%$ & 1 & 70 & $57,9 \%$ \\
\hline Com certeza & 7 & $13,0 \%$ & 12 & $18,2 \%$ & 0 & 19 & $15,7 \%$ \\
Provavelmente & 9 & $16,7 \%$ & 13 & $19,7 \%$ & 0 & 22 & $18,2 \%$ \\
Talvez & 1 & $1,9 \%$ & 4 & $6,1 \%$ & 0 & 5 & $4,1 \%$ \\
Pouco provável & 2 & $3,7 \%$ & 1 & $1,5 \%$ & 0 & 3 & $2,5 \%$ \\
Certamente não & 0 & $0,0 \%$ & 2 & $3,0 \%$ & 0 & 2 & $1,7 \%$ \\
Não quis responder & 54 & $100,0 \%$ & 66 & $100,0 \%$ & 1 & 121 & $100,0 \%$ \\
\hline Total & & & & & & & 0
\end{tabular}

Fonte: Autores.

Em relação as moedas sociais, apesar de já existirem no Brasil desde a década de 90, com o banco Palma (Mostagi et al, 2019), observou-se na pesquisa que os comerciantes não tinham qualquer informação sobre esse assunto. As moedas sociais podem auxiliar na diminuição da pobreza em uma região, pois fortalecem as transações comerciais locais. Com a pandemia, a população tefeense perdeu poder aquisitivo e a implementação de uma moeda social poderia ajudar principalmente os pequenos comerciantes a realizar suas transações comerciais. Rigo e colaboradores (2019) afirmam que para compreender a importância de uma moeda social faz-se necessário inseri-la no contexto de tecnologias sociais apropriadas à territórios, viabilizada por bancos comunitários, o que resulta em prestação de serviços bancários e financeiros incluindo acesso ao microcrédito. Porém, isso exige que sejam sensibilizados consumidores, prestadores de serviços, produtores e comerciantes locais para o uso e aceitação da moeda social como meio de pagamento no seu cotidiano.

\section{Conclusão}

Por meio da pesquisa de opinião na cidade de Tefé-Am, foi possível verificar como os comerciantes estão fazendo uso da internet para realizar suas vendas no comércio local, e conhecer como eles lidariam com uma possível transformação digital através do uso de moeda social e plataformas eletrônicas digitais para pagamentos durante o seu cotidiano.

Por meio das pesquisas nos 121 comércios da região, foi possível identificar que a maioria dos comerciantes ainda recebem muitos pagamentos e realizam pagamentos em dinheiro e ainda utilizam muito as casas lotéricas para realizar essas transações. No entanto, alguns comerciantes já utilizam aplicativos de celulares para realizar transações online, porém relatam insatisfação com as redes móveis que atendem a cidade.

Apesar da baixa qualidade da internet móvel em Tefé, as comerciantes do gênero feminino apresentaram interesse em anunciar seus produtos em plataformas digitais para alavancar suas vendas.

A pesquisa apresentou como a população que gere o comércio local está antenada nas novas tecnologias digitais, mas foi observada uma falta de conhecimento a respeito de moedas sociais, no entanto após explicação, notou-se interesse no uso de plataformas sociais financeiras.

Uma sugestão para trabalhos futuros seria uma pesquisa de opinião quantitativa e qualitativa com a população da cidade de Tefé - Am em geral, sobre a internet local e o uso de uma moeda social local atrelada a uma plataforma digital financeira, uma vez que seu uso poderia trazer um desenvolvimento para a região. Também seria interessante compreender como ocorre a evolução das formas de pagamento a médio e longo prazo na cidade de Tefé e como a qualidade da internet pode ter relação com essa evolução. 


\section{Referências}

Bez Fontana, A. S., Silva, F. S., Leigus, A. \& Zanini, W. A. (2018). Grau de satisfação dos clientes do sistema e-commerce localizados na cidade de Cafelândia-Pr. Administração em Foco. Segunda edição, 1-27. https://faculdadeunica.edu.br/wp-content/uploads/2019/04/ARTIGO-2.pdf

AgênciaBrasil. (2021). Sobe para 82,7\% percentual de domicílios com internet, diz IBGE. https://agenciabrasil.ebc.com.br/geral/noticia/2021-04/sobe-para827-percentual-de-domicilios-com-internet-diz-ibge

Chew, E. K. (2015). Digital Organizations of the Future. In: Collin, J., Hiekkanen, K., Korhonen, J.J., Halén, M., Itälä, T., Helenius. M. (eds). It Leadership in Transition - The Impact of Digitalization on Finnish Organizations. Helsinki. Aalto University.

Correa, R. F., Rugoni, T. C., Albuquerque, P., Camozzi, C., Souza, S. F. \& Aihara, S. (2006). O impacto do e-commerce na sociedade. Revista de Informática Aplicada, 2 (2), 94-110.

Diniz, E. H. (1999). Comércio Eletrônico: Fazendo Negócios por meio da Comércio Eletrônico: Fazendo Negócios por meio da Internet. RAC, 3 , 71-86.

Ebit|Nielsen. (2021). Webshoppers 43 ${ }^{\text {a }}$ edição. https://www.mobiletime.com.br/wp-content/uploads/2021/03/Webshoppers_43.pdf

Freire, D. A. \& Salgado, E. B. (2019). E-Commerce no Brasil: Panorama Geral e Principais Desafios. Trabalho de conclusão de curso. Rio de Janeiro: UFRJ/ Escola Politécnica.

Fowller, F. J. (2009). Survey Research Methods. Thousand Oaks. SagePublications, Inc.

Garcia, L. A., Silva, J. E. P., Katchorovski, Y. F. \& Volanin, F. (2020). Análise do E-Commerce como oportunidade promissora para a cidade de GuarapuavaPR e Região. Anais do X Congresso de Engenharia de Produção.

Guidini, P. A. A. (2018). Comunicação com o mercado por meio de aplicativos: desafios e oportunidades. Signos do Consumo, São Paulo, 10 (1), 59-69. doi: https://doi.org/10.11606/issn.1984-5057.v10i1p59-69

Hair, J. F. JR, Wolfinbarger, M., Money, A. H., Somouel, P. \& Page, M. J. (2003). Essentials of business research methods. Nova Jersey. John Wiley \& Sons. Instituto Brasileiro de Geografia e Estatística (IBGE). (2021). Tefé, Amazonas, IBGE Cidades. https://cidades.ibge.gov.br/brasil/am/tefe/panorama

Lima, H. \& Francisco, E. R. (2021). Transformação Digital: Revolução Nos Meios De Pagamento Digitais. Gvexecutivo. 20 (1). $23-25$.

Mostagi N. C., Pires, L. L., Mahnic, C. L. P. \& Santos, L. M. L. (2019). Banco Palmas: inclusão e desenvolvimento local. Interações, Campo Grande-MS, 20 (1), 111-124.

Okano, M. T., Simões, E. A. \& Langhi, C. (2019). Plataformas de negócios digitais: o poder da transformação digital nos dispositivos moveis. Research, Society and Development, 9 (2), e95922097.

Pinsonneault, A. K. \& Kenneth, L. (1993). Survey Research Methodology in Management Information Systems: An Assessment. Journal of management information. https://escholarship.org/uc/item/6cs4s5f0

Premebida, E. A. (2021). E-commerce em 2020, um cenário de oportunidades em meio a pandemia. Research, Society and Development, 10 (2), 1-7.

Ribeiro, R. C. (1996). Viagens à “corte do Solimões”- Manaus. Gráfica REX.

Rigo, A. S., Cançado, A. C. \& Silva Junior, J. T. (2019). Desafios e potencialidades das moedas complementares: explorando sua utilização e significado para o desenvolvimento. Revista Tecnologia Sociedade, 15 (38), 303-321.

Rodrigues, E. A. (2011). Rede urbana do Amazonas: Tefé como cidade média de responsabilidade territorial na calha do médio Solimões. Dissertação de Mestrado. Universidade Federal do Amazonas-UFAM.

Santos, R. N. F. (2012). Lugar e representação: um estudo sobre o bairro do Abial no município de Tefé/Am. Dissertação de Mestrado. Universidade Federal Do Amazonas - UFAM.

Sebrae (2017). E-book: Informe de mercado e-commerce. EAD Sebrae.

Viana, J. (2014). A evolução do e-commerce no Brasil e as perspectivas desse mercado para o futuro. Mundo e Carreira. https://mundocarreira.com.br/mercado-de-trabalho/evolucao-e-commerce-brasil-e-perspectivas-desse-mercado-para-o-futuro/

Vieira, S. (2016). Introdução à bioestatística, Elsevier Editora LTDA.

Yoshida, L. P. (2015). Uma Análise Das Vantagens E Desvantagens Dos Portais De compras Coletivas Sob A Ótica Do Empreendedor: Um Estudo MultiCasos em MPE’s do Setor de Serviços. Trabalho de Conclusão de Curso. Faculdade Campo Limpo Paulista - FACCAMP. 